\title{
An Innovative Architecture of UTC GPS/INS System with Improved Performance under Severe Jamming
}

\author{
Xueyun Wang, Jingjuan Zhang, Wei Wang, and Pengyu Gao \\ School of Instrument Science and Opto-Electronics Engineering, Beihang University, Beijing 100191, China \\ Correspondence should be addressed to Jingjuan Zhang; jingjuan1002@sina.com
}

Received 17 January 2014; Accepted 12 February 2014; Published 19 March 2014

Academic Editor: Guanghui Wen

Copyright ( 2014 Xueyun Wang et al. This is an open access article distributed under the Creative Commons Attribution License, which permits unrestricted use, distribution, and reproduction in any medium, provided the original work is properly cited.

\begin{abstract}
Ultratightly coupled (UTC) architecture is believed to be the best architecture for Global Positioning System (GPS) and Inertial Navigation System (INS) integration system due to the advanced data fusion strategy and effective mutual assistance between the subsystems. However the performance of UTC GPS/INS system will be degraded by severe jamming interference, especially when low-grade inertial measurement unit (IMU) is used. To solve this problem an innovative architecture of UTC GPS/INS system is proposed. Since GPS receiver's antijamming ability is closely related to tracking loop bandwidth, adaptive tracking loop bandwidth based on the fuzzy logics is proposed to enhance antijamming ability for GPS receiver. The bandwidth will be adapted through a fuzzy logic controller according to the calculated carrier to noise intensity ratio $(C / N 0)$. Moreover, fuzzy adaptive integration Kalman filter (IKF) is developed to improve estimation accuracy of IKF when measurement noises change. A simulation platform is established to evaluate the innovative architecture and results demonstrate that the proposed scheme improves navigation performance significantly under severe jamming conditions.
\end{abstract}

\section{Introduction}

GPS/INS system is the best solution for many navigation applications and has been a research hotspot since the day it was proposed [1-3]. By the integration level, integration systems can be classified into three types: loosely, tightly, and ultratightly (also called deeply). Authors differ on what exactly constitutes each of the three couplings [4-6]; the definitions used here are closest to those given by Gao in [5]. If the GPS receiver is assisted by INS, the GPS/INS system is defined as ultratightly coupled system, in which either pseudorange (PR)/pseudorange-rate (PRR) or inphase $(I)$ /quadrature $(Q)$ are taken as measurements by IKF. Due to the advanced data fusion strategy and enhanced navigation performance, UTC GPS/INS system becomes a superior solution to robust and precise navigation in severe jamming environments. However when low-grade inertial sensors (i.e., gyroscopes and accelerometers), like microelectromechanical system (MEMS) devices, are used in UTC GPS/INS system, the tracking loop bandwidth of GPS has to be wide enough to calibrate sensor errors effectively [7], which weakens the jamming resistance ability at the same time.
Therefore the antijamming property is an important issue for MEMS-based UTC GPS/INS system.

Some investigations have been conducted on this subject. Gao proposed another new architecture of UTC system with a cooperated tracking loop (COOP) inside of traditional GPS tracking loops [5]. A method that adapts bandwidth of delay locked loop (DLL) to carrier to noise intensity ratio $(C / N 0)$ was proposed to handle high interference or weak signal environment and it indicated improvement in antijam margin over an INS/GPS with fixed bandwidths [7], but carrier tracking loop, that is, phase locked loop (PLL), is not considered. Besides the adaptation is realized by a quadratic polynomial which is derived by experiments and experiences. Ohlmeyer proposed a UTC GPS/INS system featured by a bank of prefilters to estimate code delay error and the Doppler frequency error for each satellite. This design was also considered more robust to jamming and dynamics [8]. Effects of inertial measurement unit (IMU) on weak signal tracking were also analyzed. Research was conducted on performance enhancement for ultratightly coupled GPS/INS system using a fuzzy adaptive strong tracking unscented Kalman filter, verifying the effectiveness of fuzzy logic adaptive control 
(FLAC) [9]. Similar conclusions about advantages of FLAC were also drawn in [10-12].

Aimed at improving navigation performance under severe jamming and changing noise conditions, an innovative architecture of UTC GPS/INS system is proposed. Firstly the FLAC is applied to adjust PLL bandwidth according to GPS signal noise intensity which is measured by $\mathrm{C} / \mathrm{N} 0$. Adaptive PLL bandwidth will enhance the antijamming ability of the GPS receiver. Moreover FLAC is also used in the design of adaptive IKF in which the measurement noise model is tuned online according to the innovation covariance. The state estimation accuracy of adaptive IKF is improved if the measurement noises change. The proposed architecture is assessed by simulations conducted through a self-developed simulation platform.

\section{Problem Description}

As PLL endures most of the noise interference and dynamic stress, DLL is generally locked in if PLL is locked in, so most attention is paid to the vulnerable PLL tracking. For robust and precise navigation, PLL should always be locked in. Generally severe jamming and high dynamics are the causes that force PLL to lose track. Narrowing the tracking loop bandwidth will improve the antijamming ability but will lose track more easily under high dynamics due to the large Doppler shifting caused by vehicle maneuver. In UTC GPS/INS system, as most of the Doppler shifting is compensated by INS aiding information, PLL tracking loops only need to eliminate the residual Doppler shifting mainly caused by errors of INS aiding information. The contradictory requirement for tracking loop bandwidth is much relieved. However, when low-grade MEMS IMU is used, the errors of aiding the Doppler information are considerable. Therefore, PLL bandwidth has to be wide enough to handle aiding errors and calibrate MEMS sensors as well. To obtain both good high-dynamic property and strong antijamming ability, PLL bandwidth needs to be adapted according to the noise intensity. Nevertheless, although adaptive bandwidth is employed, severe jamming could still cause obvious changes to the characteristics of GPS outputs (i.e., IKF measurements), so fixed measurement noise model for IKF described a priori is inappropriate. Hence an adaptive IKF in which measurement noise covariance matrix can be tuned in realtime is needed.

\section{Methodology}

3.1. Fuzzy Logic Adaptive PLL Bandwidth. When PLL is well locked in, the errors of PLL-derived pseudorange-rate are mainly thermal noise whose standard deviation is affected by its bandwidth and $C / N 0$. The relationship can be expressed as follows $[13,14]$ :

$$
\operatorname{std}(\dot{\rho})=\frac{c}{\sqrt{2} \pi f_{L} T_{D}} \sqrt{\frac{B_{n}}{C / N 0}\left[1+\frac{1}{2 T_{\mathrm{coh}} \cdot C / N 0}\right]},
$$

where $\operatorname{std}(\dot{\rho})$ is standard deviation of PRR, $c$ is light speed, $f_{L}$ is carrier frequency, $T_{D}$ is the Doppler integration time,

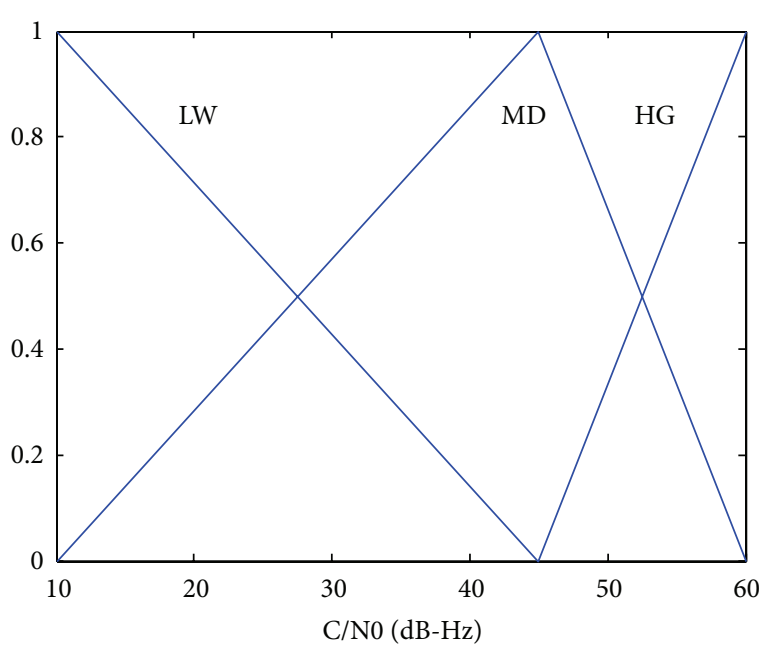

Figure 1: C/N0 fuzzification.

TABLE 1: Fuzzy rules for adaptive PLL bandwidth.

\begin{tabular}{lc}
\hline If $C / N 0$ is & Then bandwidth is \\
\hline Low (LW) & Narrow (NR) \\
Medium (MD) & Normal (NM) \\
High (HG) & Wide (WD) \\
\hline
\end{tabular}

$T_{\text {coh }}$ is coherent integration time for $I$ and $Q$, and $B_{n}$ is PLL bandwidth.

All the parameters except for $B_{n}$ and $C / N 0$ are constants for a specific GPS receiver, so if $C / N 0$ decreases, $B_{n}$ has to decrease accordingly to constrain PRR error within a certain level. If conversely $C / N 0$ increases, $B_{n}$ ought to increase to obtain better performance under high dynamics and better calibration for inertial sensors. However, in fact the bandwidth should not be adjusted thoroughly according to (1). The first reason is that PLL are not always well locked in. Secondly, the corresponding bandwidth in severe jamming could be too much narrow that PLL easily loses track due to vehicle maneuvers or errors of INS aiding information. As basic adaptation principles are clear, fuzzy adaptive logic control is preferred for the adjustment of PLL bandwidth.

On the basis of the analyses above an adaptive PLL bandwidth based on fuzzy logic is designed. The fuzzy logic controller's input is $\mathrm{C} / \mathrm{NO}$ (in unit of $\mathrm{dB}-\mathrm{Hz}$ ) and output is PLL bandwidth (in unit of $\mathrm{Hz}$ ). The membership function (MF) for fuzzification and defuzzification is shown in Figures 1 and 2 . Note that the bandwidth starts at $0.1 \mathrm{~Hz}$, not 0 . Fuzzy rules are listed in Table 1.

The designed fuzzy logic controller belongs to the type of Mamdani. Max-Min inference method is applied to define the results of fuzzy rules and centroid defuzzification is employed to extract outputs.

A vital issue arises on how to determine the input, $C / N 0$. Here variance summing method (VSM) is used to calculate $\mathrm{C} / \mathrm{NO}$ using the sampled GPS signals after downconverting $[15,16]$. 


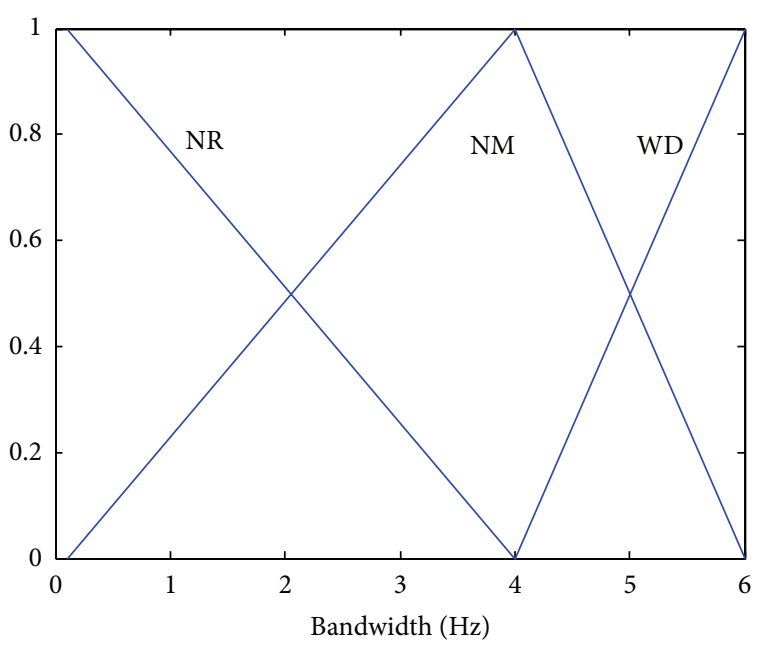

FIgURE 2: PLL bandwidth defuzzification.

The integrate-and-dump results at sampled time $i$, inphase $I_{i}$ and quadrature $Q_{i}$, can be divided into signal and noise:

$$
\begin{gathered}
I_{i}=I_{i}^{s}+I_{i}^{n}=\frac{A M_{E} D_{i}}{\sqrt{2}} \cdot \cos \left(\delta \varphi_{i}\right)+I_{i}^{n}, \\
Q_{i}=Q_{i}^{s}+Q_{i}^{n}=\frac{A M_{E} D_{i}}{\sqrt{2}} \cdot \sin \left(\delta \varphi_{i}\right)+Q_{i}^{n},
\end{gathered}
$$

where $A$ and $D_{i}$ are signal amplitude and navigation data, respectively, and $\delta \varphi_{i}$ denotes PLL phase error. $M_{E}$ is the integration number yielding $M_{E}=T_{\text {Int }} / T_{s}$ where $T_{\text {Int }}$ is coherent integration interval and $T_{s}$ is sample interval.

If PLL is in good lock-in condition, which means $\delta \varphi_{i}$ is fairly small, $I_{i}$ and $Q_{i}$ approximate to

$$
\begin{gathered}
I_{i}=\frac{A M_{E} D_{i}}{\sqrt{2}}+I_{i}^{n}, \\
Q_{i}=Q_{i}^{n} .
\end{gathered}
$$

Since binary phase shift keying (BPSK) technique is used in GPS signal modulation, navigation data yields $D_{i}= \pm 1$. Set a new variable by the following equation:

$$
\begin{aligned}
Z_{i}= & I_{i}^{2}+Q_{i}^{2}=\left(\frac{A M_{E} D_{i}}{\sqrt{2}}\right)^{2} \\
& +\frac{A M_{E} D_{i}}{\sqrt{2}} \cdot I_{i}^{n}+\left(I_{i}^{n}\right)^{2}+\left(Q_{i}^{n}\right)^{2} .
\end{aligned}
$$

Assume $I_{i}^{n}$ and $Q_{i}^{n}$ are both zero-mean noise and have the same standard deviation $\delta_{i q}$, so the mean and variance of $Z_{i}$ are as follows, respectively:

$$
\begin{gathered}
\bar{z}=\frac{\left(A M_{E}\right)^{2}}{2}+2 \delta_{i q}^{2}, \\
\delta_{z}^{2}=2\left(A M_{E}\right)^{2} \delta_{i q}^{2}+4 \delta_{i q}^{4} .
\end{gathered}
$$

Thus equations of the average carrier power $P_{c}$ and average noise power $P_{n}$ take the following forms:

$$
\begin{aligned}
& P_{c}=\frac{\left(A M_{E}\right)^{2}}{2}=\sqrt{(\bar{z})^{2}-\delta_{z}^{2}}, \\
& P_{n}=2 \delta_{i q}^{2}=\bar{z}-\sqrt{(\bar{z})^{2}-\delta_{z}^{2}} .
\end{aligned}
$$

Finally the carrier to noise density ratio $\mathrm{C} / \mathrm{NO}$ can be determined by the following formula:

$$
\begin{aligned}
\frac{C}{N_{0}} & =10 \log _{10}\left(\frac{P_{c}}{P_{n}} \cdot \frac{1}{T_{\text {Int }}}\right) \\
& =10 \log _{10}\left(\frac{\sqrt{(\bar{z})^{2}-\delta_{z_{c}}^{2}}}{\bar{z}-\sqrt{(\bar{z})^{2}-\delta_{z}^{2}}} \cdot \frac{1}{T_{\text {Int }}}\right) .
\end{aligned}
$$

3.2. Fuzzy Logic Adaptive IKF. Details about standard algorithm of the Kalman filter can be found in many papers [17]. Applications based on KF not only demand correct state transition matrix and measurement matrix but also presume complete and accurate a priori information about the process noise and measurement noise. If any of the requirements are not met, KF may propagate suboptimal estimates or even diverge. Previously substantial work has been done to obtain correct state transition matrix and measurement matrix for GPS/INS system, making it not a problem at all. Moreover, the stochastic statistics of the process noise, which mainly refers to inertial sensors' white noise and the driving noise (also white) of the Gauss-Markov process, can be determined with methods such as the Allen variance. Besides, characteristics of process noises do not change greatly unless faults happen. However it is different for the measurement noises. The covariance of measurement noise cannot be constrained within a certain level under various jamming conditions, even though PLL bandwidth has been adapted. Once measurement noise changes, its covariance matrix needs to be corrected so that divergence caused by noise model inaccuracy could be avoided and better state estimates could be made. That is the exact purpose of adaptive IKF. There are several ways to accomplish this goal and they are classified into four categories, namely, Bayesian, maximum likelihood, correlation (autocorrelation), and covariance matching [18]. The fourth approach implemented by fuzzy logic control is selected for the innovative UTC GPS/INS system and the algorithms are as follows.

Innovation, also named as residual, is critical information for the Kalman filter. It is defined as the discrepancy between actual measurements and predicted measurements:

$$
e_{k}=z_{k}-H_{k} x_{k}^{-} .
$$

In the equation, $k$ is the time sequence mark and $z_{k}$ represents the actual measurements. $H_{k}$ is the measurement matrix and $x_{k}^{-}$is the formerly estimated state vector. Theoretically in normal conditions, the innovation is zero-mean white noise 
TABLE 2: Fuzzy rules for adaptive IKF.

\begin{tabular}{lc}
\hline If covariance ratio is & Then scale factor is \\
\hline Low (LW) & Smaller (SM) \\
Medium (MD) & Normal (NM) \\
High (HG) & Bigger (BG) \\
\hline
\end{tabular}

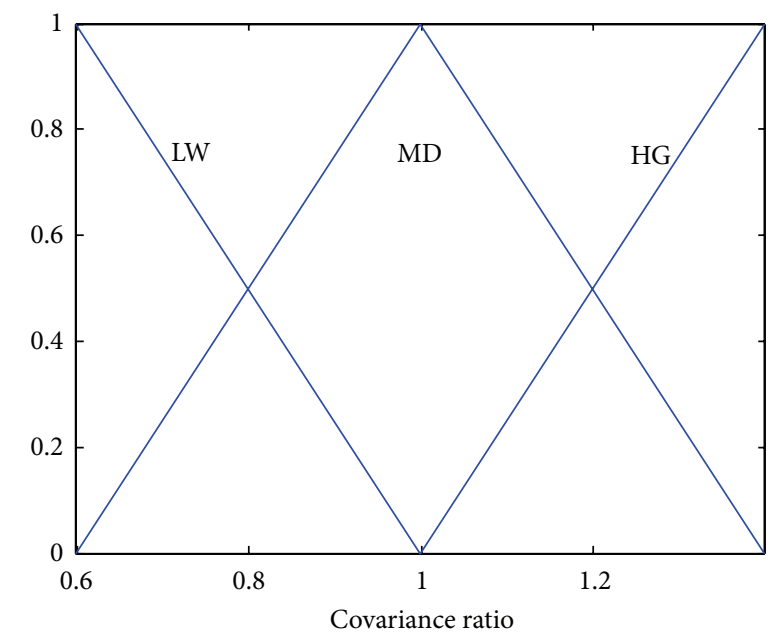

FIGURE 3: Fuzzification for covariance ratio.

whose covariance is related to the covariance of process noise and the measurement noise, yielding

$$
C_{e_{k}}=H_{k}\left(F_{k / k-1} P_{k-1} F_{k / k-1}^{T}+Q_{k-1}\right) H_{k}^{T}+R_{k-1},
$$

where $F_{k / k-1}$ is state transition matrix from time $k-1$ to $k$, $P_{k-1}$ is covariance matrix of estimation errors at time $k-1$, $Q_{k-1}$ is covariance matrix of process noise, $R_{k-1}$ is covariance matrix of measurement noise, and $Q_{k-1}$ and $R_{k-1}$ are set as diagonal matrices by the assumption that all the process noises and measurement noises are uncorrelated.

The actual innovation covariance is defined as the averaging covariance of the $N$ newest sampled innovations and the number $N$ is decided by the length of moving window:

$$
T_{e_{k}}=\frac{1}{N} \sum_{i=i_{0}}^{k} e_{i} \cdot e_{i}^{T},
$$

where $i_{0}=k-N+1$ is the start of the moving window. $N$ is applied empirically to provide smoothing and the exact value employed in this paper is 10 .

If $\mathrm{KF}$ proceeds normally, the theoretical and actual covariance of innovations should be identical; otherwise the covariance matrix of measurement noise $R_{k-1}$ must be impropriate provided that $H_{k}, F_{k / k-1}$, and $Q_{k-1}$ are all correct. So the fundamental principle of fuzzy logic adaptive IKF is to adjust measurement noise covariance matrix as what it should be according to the discrepancy between the theoretical and actual covariance of innovations.

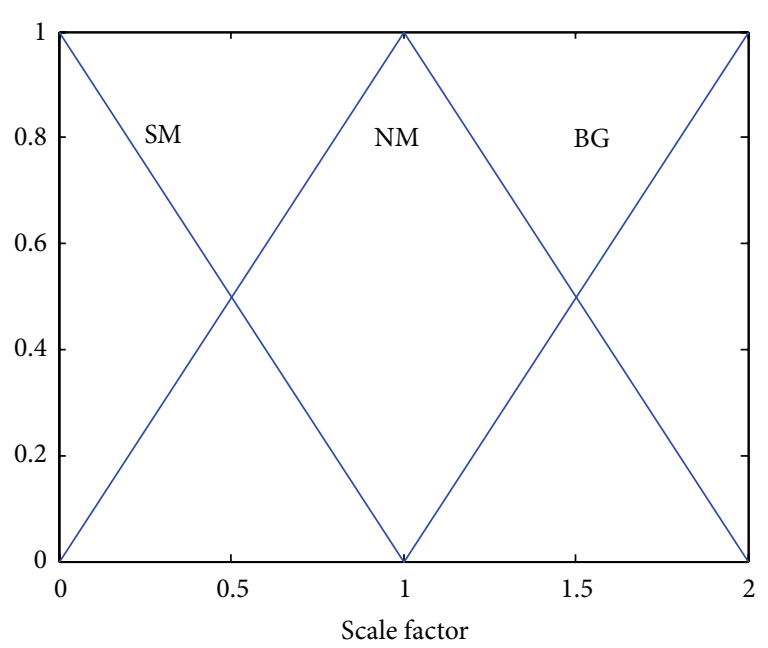

Figure 4: Defuzzification for scale factor.

The input for the fuzzy logic controller is the ratio of the actual innovation covariance and theoretical innovation covariance:

$$
\alpha_{k}=\operatorname{diagonal}\left(\frac{C_{e_{k}}}{T_{e_{k}}}\right) \text {. }
$$

Only the diagonal elements are reserved to be the inputs. As the fuzzy logic controller is a single-input-single-output system, each of the diagonal elements in $\alpha_{k}$ has to go through the fuzzy logic controller one after another to achieve a corresponding result. Finally all the results will form the controller's output, which is also a diagonal matrix containing scale factors for the correction of measurement noise covariance matrix. For the next integration navigation, the corrected $R_{k}$ is shown as follows:

$$
R_{k}=\beta_{k} \cdot R_{k-1},
$$

where $\beta_{k}$ is the scale factor matrix.

The input and output MFs of the fuzzy logic controller for adaptive IKF are shown in Figures 3 and 4. Fuzzy rules are listed in Table 2. All configurations about the fuzzy logic controller are identical with those of PLL bandwidth.

Since fuzzy logic adaptive controller is designed and applied to improve the antijamming ability of IKF, the mathematic model of the IKF in this innovative architecture is different from conventional IKF. The mathematic model of new IKF can be divided into two parts and they are detailed as follows. 


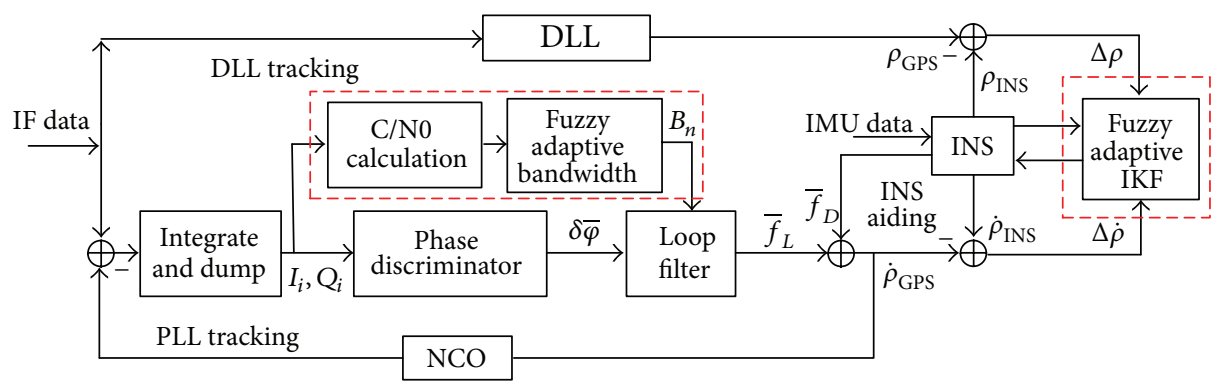

FIGURE 5: Scheme diagram of the innovative architecture of the UTC GPS/INS system.

Part 1. The algorisms of the Kalman filter are

$$
\begin{gathered}
K_{k}=P_{k}^{-} H_{k}^{T}\left(H_{k} P_{k}^{-} H_{k}^{T}+R_{k}\right), \\
e_{k}=z_{k}-H_{k} x_{k}^{-}, \\
x_{k}=x_{k}^{-}+K_{k} e_{k}, \\
x_{k+1}^{-}=\Phi_{k} x_{k}, \\
P_{k}=\left(I-K_{k} H_{k}\right) P_{k}^{-}, \\
P_{k+1}^{-}=F_{k+1 / k} P_{k} F_{k+1 / k}^{T}+Q_{k} .
\end{gathered}
$$

The meanings of the symbols that are not explained in this paper could be found in [17].

Part 2. The adjustment of measurement covariance matrix is

$$
\begin{gathered}
C_{e_{k}}=H_{k} P_{k+1}^{-} H_{k}^{T}+R_{k}, \\
T_{e_{k}}=\frac{1}{N} \sum_{i=i_{0}}^{k} e_{i} \cdot e_{i}^{T}, \\
\alpha_{k}=\operatorname{diagonal}\left(\frac{C_{e_{k}}}{T_{e_{k}}}\right), \\
\beta_{k+1}=\operatorname{FLAC}\left(\alpha_{k}\right), \\
R_{k+1}=\beta_{k+1} \cdot R_{k} .
\end{gathered}
$$

After the adjustment of measurement covariance matrix, algorisms of KF will be conducted again with the new measurement covariance matrix. Through correcting $R_{k}$ the fuzzy logic controller improves the antijamming ability of the conventional IKF so that the UTC INS/GPS system gains a much better performance under severe jamming conditions.

The scheme diagram of the innovative architecture of UTC GPS/INS system is shown in Figure 5.

\section{Simulation and Analysis}

4.1. Simulation Configurations. To verify the effectiveness of the proposed architecture, a simulation package is developed

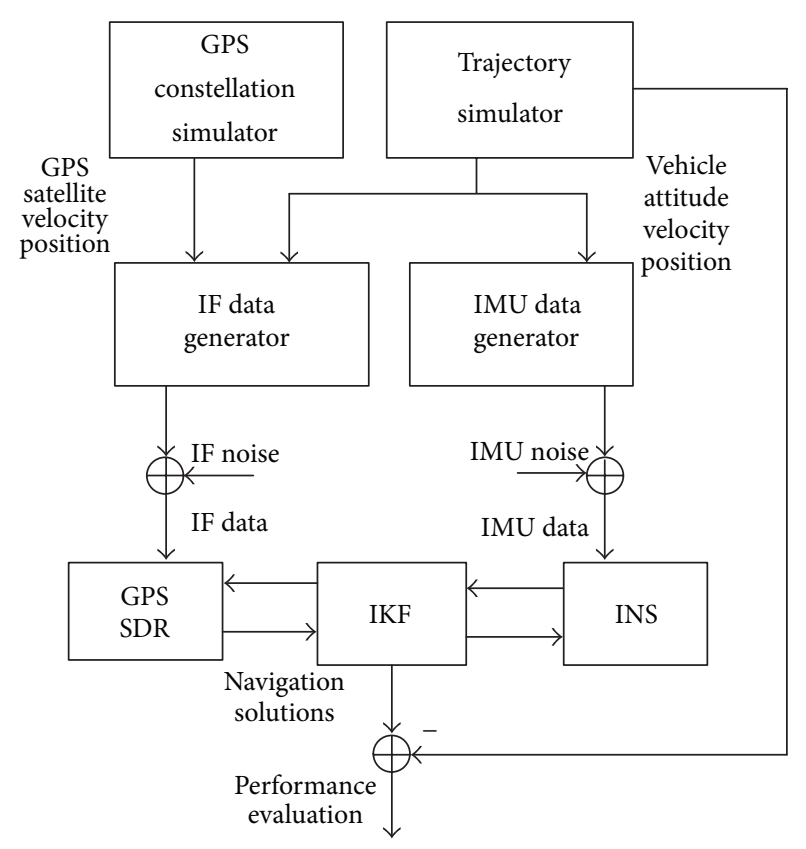

FIGURE 6: Simulation platform compositions.

and its compositions are shown in Figure 6. First of all, the trajectory simulator (TS) gives a specific trajectory which includes vehicle attitude, velocity, and positions, based on which the IMU data generator calculates the actual acceleration and all the angular rates for the inertial sensor triads in body frame. Secondly the intermediate frequency (IF) data generator takes the actual trajectory from TS, as well as all kinematics parameters of GPS satellites from the GPS constellation simulator, to produce the IF data for GPS software defined receiver (SDR). At last IKF takes PR and PRR from GPS SDR and navigation data from INS to perform integration navigation. The innovative scheme will be evaluated by comparing its navigation solutions with the actual trajectory. The GPS SDR is developed on the basis of the fundamental work previously done by Kai Borre and so forth [19] and it has been verified by processing real GPS IF data to obtain correct navigation results. The IF data generator and GPS constellation simulator are then certified by the verified GPS SDR. 


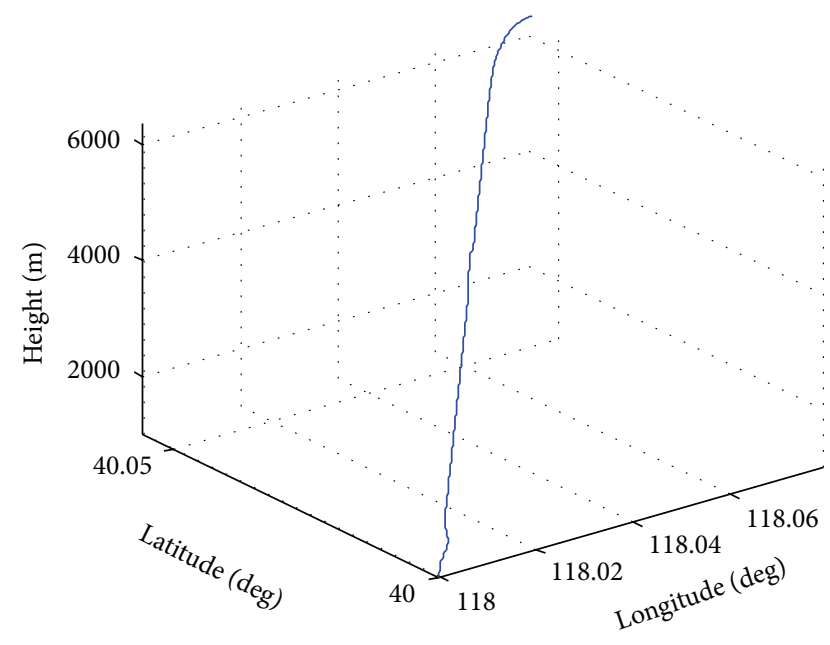

FIgURE 7: 3-dimensional position.

The 3-dimensional position of the 50-second simulated trajectory is shown in Figure 7 and magnitudes of velocity and acceleration are shown in Figure 8.

A four-satellite GPS constellation with a nominal GDOP of 2.52 is used by the single frequency SDR whose integrateand-dump operation rate and PR/PRR update rate are both $1000 \mathrm{~Hz}$. Second-order tracking loops are engaged for DLL and PLL. The bandwidth of DLL is $0.1 \mathrm{~Hz}$ while the bandwidth of PLL will be discussed specially in the next section. The error characteristics of IMU are basically determined in accordance with generic MEMS sensors and the essential parameters are listed in Table 3. The IMU data sampling interval is 1 millisecond while the integrated navigation is operated every 100 milliseconds. Although Earth-CenteredEarth-Fixed (ECEF) coordinates are used to calculate PR and PRR and so forth, navigation results are finally provided in geographic coordinates. As each satellite channel is independent and equivalent, only the loop tracking and PRR results of satellite number 3 (pseudorandom number 3 ) are displayed to draw conclusions in the following sections.

4.2. Evaluation of the Proposed Architecture. A wide range of jamming is artificially simulated from $20 \mathrm{~s}$ to $38 \mathrm{~s}$ to evaluate the performance of fuzzy adaptive PLL bandwidth and fuzzy adaptive IKF. Jamming forces $C / N 0$ to decrease sharply from $45 \mathrm{~dB}-\mathrm{Hz}$ to $25 \mathrm{~dB}-\mathrm{Hz}$ at $20 \mathrm{~s}$ and keeps it at $25 \mathrm{~dB}-\mathrm{Hz}$ until $30 \mathrm{~s}$. Then jamming gradually weakens and $\mathrm{C} / \mathrm{NO}$ recovers to $45 \mathrm{~dB}-\mathrm{Hz}$ at an increment of $2.5 \mathrm{~Hz}-\mathrm{dB}$ every second. The true $C / N 0$, calculated $C / N 0$, and the adapted PLL bandwidth are shown in Figure 9 . For standard UTC GPS/INS system the PLL bandwidth is fixed at $3 \mathrm{~Hz}$. However for the innovative one the bandwidth is adapted down to about $0.8 \mathrm{~Hz}$ according to the calculated $\mathrm{C} / \mathrm{N} 0$ whose accuracy is better than $3 \mathrm{~dB}-\mathrm{Hz}$. As a result of the narrowed PLL bandwidth, the PRR accuracy of innovative architecture has improved significantly compared with the standard one, from $3.02 \mathrm{~m} / \mathrm{s}$ to $0.85 \mathrm{~m} / \mathrm{s}$ in terms of maximum error as shown in Figure 10. It is worth pointing out that if the PLL bandwidth of standard architecture is set at $0.8 \mathrm{~Hz}$,
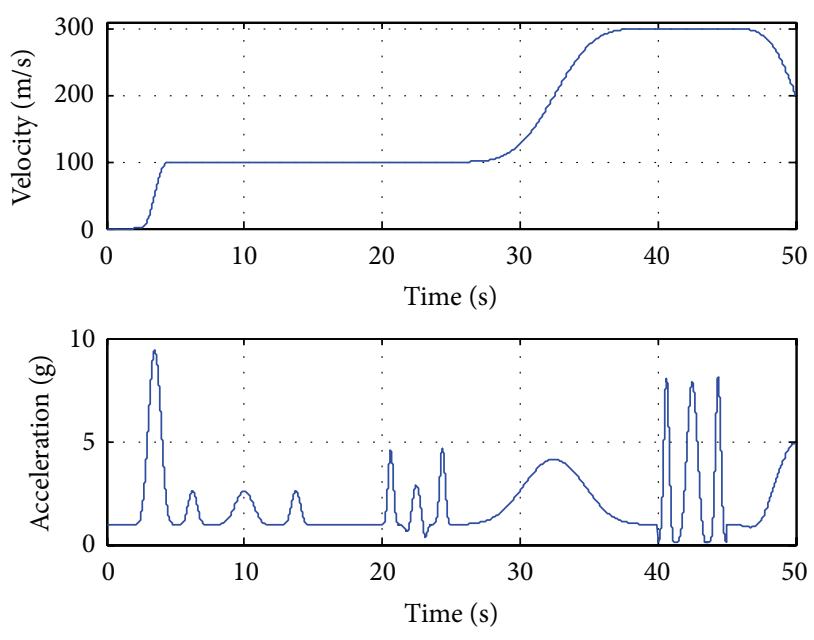

Figure 8: Acceleration and velocity.

TABLE 3: IMU error characteristics.

\begin{tabular}{lcc}
\hline \multirow{2}{*}{ Error sources } & \multicolumn{2}{c}{ Error values } \\
& Gyroscopes & Accelerometers \\
\hline Bias & $60 \mathrm{deg} / \mathrm{h}$ & $10 \mathrm{mg}$ \\
Scale factor & $200 \mathrm{ppm}$ & $200 \mathrm{ppm}$ \\
Random walk & $0.2 \mathrm{deg} / \sqrt{\mathrm{h}}$ & $0.12 \mathrm{~m} / \mathrm{s} / \sqrt{\mathrm{h}}$ \\
\hline
\end{tabular}

PLL will lose track during the initial speedup because the high acceleration causes great dynamic pressure that the narrowed bandwidth is not able to handle. Therefore, the fuzzy adaptive PLL bandwidth is a more intelligent and robust solution for severe jamming interference.

Nevertheless, in spite of being improved, the PRR noise still increases compared with that when jamming is not involved, from $0.10 \mathrm{~m} / \mathrm{s}$ to $0.23 \mathrm{~m} / \mathrm{s}$ in terms of standard deviation, so the covariance matrix of measurement noise should be corrected accordingly. The PRR noise standard deviations that are used to form the measurement noise covariance matrices of standard IKF and innovative one are shown, respectively, in the upper plot of Figure 11. The ratios of real and theoretical innovation variances of satellite number 3 are shown in unit of $\mathrm{dB}$ in the lower plot of Figure 11. They indicate how well the measurement noise model corresponds to reality. In normal situations, the ratios should be around $1(0 \mathrm{~dB})$. At first the PRR noise standard deviations of both architectures are set as $0.06 \mathrm{~m} / \mathrm{s}$, which is a little bit lower than the true value $0.10 \mathrm{~m} / \mathrm{s}$. The measurement noise model of standard IKF is not modified. However, in the adaptive IKF, the PRR noise standard deviation is quickly adjusted by the IKF fuzzy logic controller to about $0.1 \mathrm{~m} / \mathrm{s}$ until the jamming happens. After jamming disappears at $38 \mathrm{~s}$, PRR noise model is tuned back to $0.1 \mathrm{~m} / \mathrm{s}$ again. For the standard architecture, the ratio of real and theoretical innovation variance ranges from $-5 \mathrm{~dB}$ to $15 \mathrm{~dB}$, but for the innovative architecture, the ratio is kept around $0 \mathrm{~dB}$ all the time, implying that the measurement noise model is correct and accurate. 


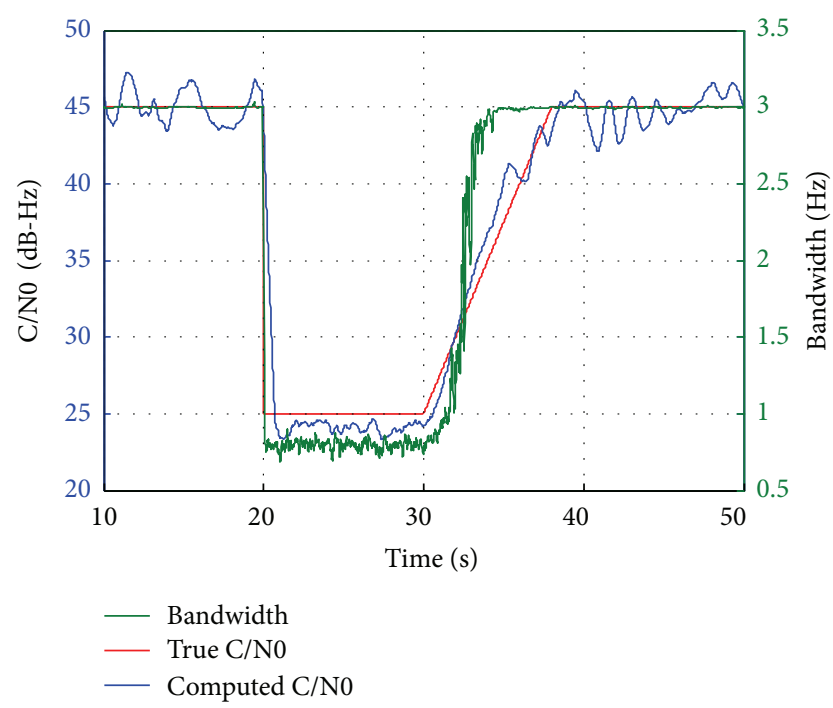

Figure 9: C/N0 and adapted bandwidth.

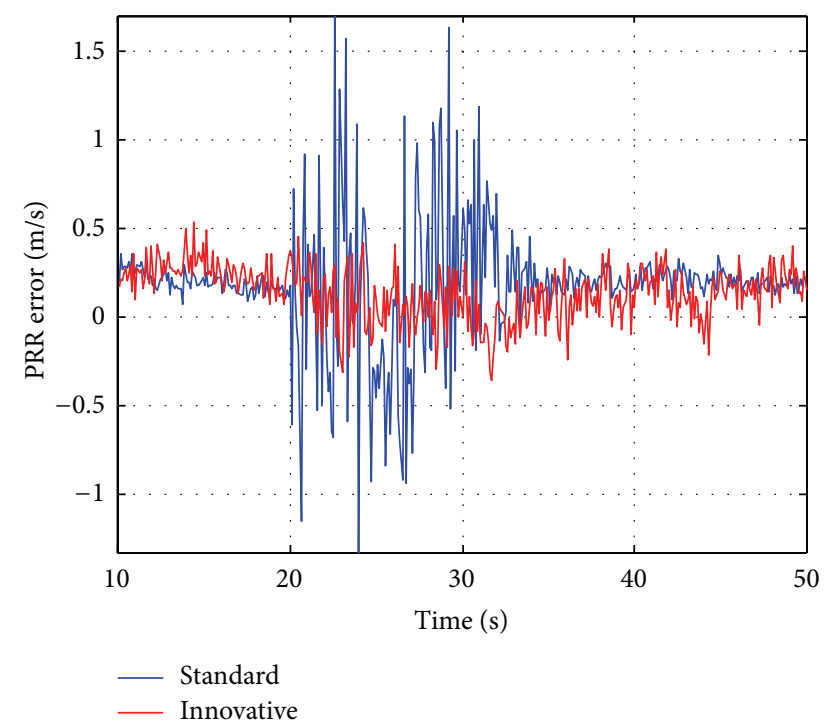

FIGURE 10: PRR error comparison in jamming.

The east, north, and up velocity errors of both architectures are given in Figure 12. Clearly the innovative architecture has a much improved navigation performance under severe jamming conditions than the standard one. In terms of maximum error, the velocity accuracy of the innovative architecture during $20 \mathrm{~s}-38 \mathrm{~s}$ is $0.73 \mathrm{~m} / \mathrm{s}$ while the standard one only gets to $2.05 \mathrm{~m} / \mathrm{s}$. In terms of standard deviation, the performances of innovative and standard schemes are $0.10 \mathrm{~m} / \mathrm{s}$ and $0.31 \mathrm{~m} / \mathrm{s}$, respectively, during the same period of time.

\section{Conclusion}

An innovative architecture of UTC GPS/INS integrated system is proposed to reinforce the antijamming ability. The architectural features include adaptive PLL bandwidth
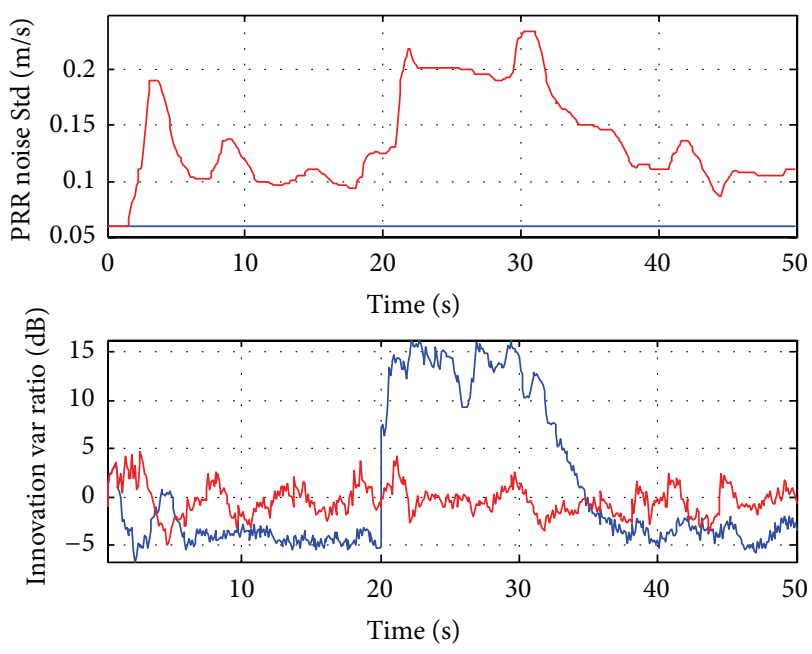

- Standard

— Innovative

FIgURE 11: Performance of adaptive and standard IKFs.
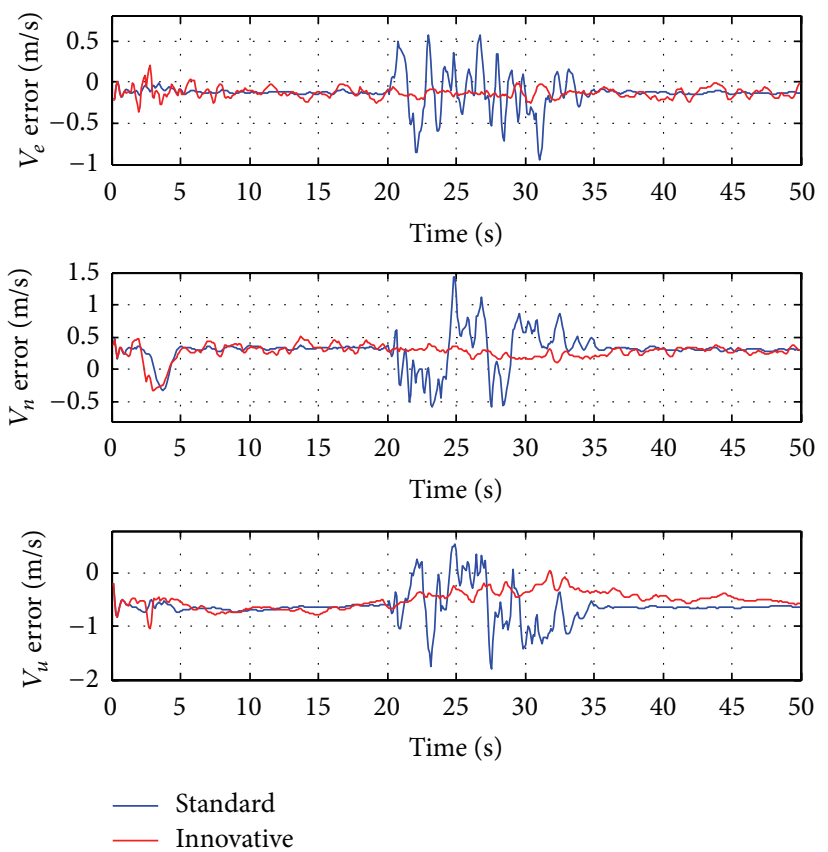

FIGURE 12: Velocity error comparisons.

and adaptive IKF, both of which are implemented through fuzzy logics. Firstly, PLL bandwidth is adjusted according to calculated $C / N 0$, so the antijamming ability of GPS receiver is reinforced. Secondly the measurement noise covariance matrix of IKF is tuned online according to innovation covariance, so the estimation accuracy is improved when the measurement noises change. The algorithms are detailed and a simulation platform is developed to verify the effectiveness of the proposed architecture. Results presented in this paper demonstrate that the innovative architecture improves the navigation performance significantly under severe jamming 
conditions. Further work essentially lies in the algorithm verification with real data and the implementation of the system by hardware. By then the practical usage of fuzzy logic and additional computation burden should be dealt with dedicatedly.

\section{Conflict of Interests}

The authors declare that there is no conflict of interests regarding the publication of this paper.

\section{Acknowledgment}

This research was sponsored by the National Natural Science Foundation of China (Grant no. 61079017). The authors are grateful for the support it provided.

\section{References}

[1] L. Semeniuk and A. Noureldin, "Bridging GPS outages using neural network estimates of INS position and velocity errors," Measurement Science and Technology, vol. 17, no. 10, pp. 27832798, 2006.

[2] Q. Zhang, X. Niu, Q. Chen, H. Zhang, and C. Shi, "Using Allan variance to evaluate the relative accuracy on different time scales of GNSS/INS systems," Measurement Science and Technology, vol. 24, Article ID 085006, 2013.

[3] L. Zhao and Q. Wang, "Design of an attitude and heading reference system based on distributed filtering for small UAV," Mathematical Problems in Engineering, vol. 2013, Article ID 498739, 8 pages, 2013.

[4] M. Lashley, D. M. Bevly, and J. Y. Hung, "Analysis of deeply integrated and tightly coupled architectures," in Proceedings of the IEEE/ION Position, Location and Navigation Symposium (PLANS '10), pp. 382-396, May 2010.

[5] G. Gao and G. Lachapelle, "A novel architecture for Ultra-Tight HSGPS-INS integration," Journal of Global Positioning System, vol. 7, pp. 46-61, 2008.

[6] D. G. Egziabher, "GNSS Solutions: weighting GNSS observations and variations of GNSS/INS integration," Inside GNSS, vol. 2, no. 1, pp. 28-33, 2007.

[7] P. D. Groves and D. C. Long, "Combating GNSS interference with advanced inertial integration," Journal of Navigation, vol. 58, no. 3, pp. 419-432, 2005.

[8] E. J. Ohlmeyer, "Analysis of an ultra-tightly coupled GPS/INS system in jamming," in Proceedings of the IEEE/ION Position, Location, and Navigation Symposium, pp. 44-53, April 2006.

[9] D. Jwo and C. Yang, "Performance enhancement for ultra-tight GPS/INS integration using a fuzzy adaptive strong tracking unscented Kalman filter," Nonlinear Dynamics, vol. 73, pp. 377395, 2013.

[10] C. Hide, T. Moore, and M. Smith, "Adaptive Kalman filtering for low-cost INS/GPS," Journal of Navigation, vol. 56, no. 1, pp. 143-152, 2003.

[11] D. Loebis, R. Sutton, J. Chudley, and W. Naeem, "Adaptive tuning of a Kalman filter via fuzzy logic for an intelligent AUV navigation system," Control Engineering Practice, vol. 12, no. 12, pp. 1531-1539, 2004.

[12] H. Bian, Z. Jin, and W. Tian, "Study on GPS attitude determination system aided INS using adaptive Kalman filter,"
Measurement Science and Technology, vol. 16, no. 10, pp. 20722079, 2005.

[13] X.-L. Wang and Y.-F. Li, "An innovative scheme for SINS/GPS ultra-tight integration system with low-grade IMU," Aerospace Science and Technology, vol. 23, pp. 452-460, 2012.

[14] R. J. Landry, "New technique to improve GPS receiver performances by acquisition and tracking threshold reduction," in Proceedings of the RTO SCi International Conference on Integrated Navigation Systems, pp. 24-26, 1999.

[15] M. L. Psiaki, O. M. Akos, and J. Thor, "A comparison of direct RF sampling and down-convert \& sampling GNSS receiver architectures," in Proceedings of the ION GPS Conference, pp. 1941-1952, 2003.

[16] M. L. Psiaki, "Block acquisition of weak GPS signals in a software receiver," in Proceedings of the 14th International Technical Meeting of the Satellite Division of the Institute of Navigation (ION GPS '01), pp. 2838-2850, 2001.

[17] M. Liu and F. Xiong, "A fuzzy adaptive GPS/INS integrated navigation algorithm," Procedia Engineering, vol. 15, pp. 660664, 2011.

[18] J. Ali, "Strapdown inertial navigation system/astronavigation system data synthesis using innovation-based fuzzy adaptive Kalman filtering," IET Science, Measurement and Technology, vol. 4, no. 5, pp. 246-255, 2010.

[19] K. Borre, D. M. Akos, N. Bertelsen, P. Rinder, and S. H. Jensen, A Software-Defined GPS and Galileo Receiver: A Single-Frequency Approach, Springer, Washington, DC, USA, 2006. 


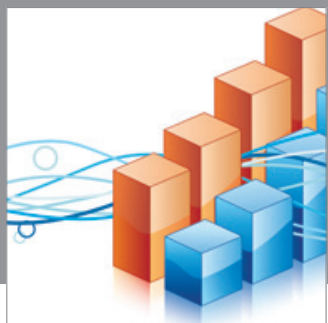

Advances in

Operations Research

mansans

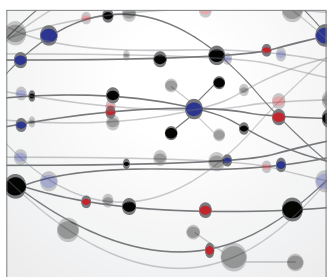

The Scientific World Journal
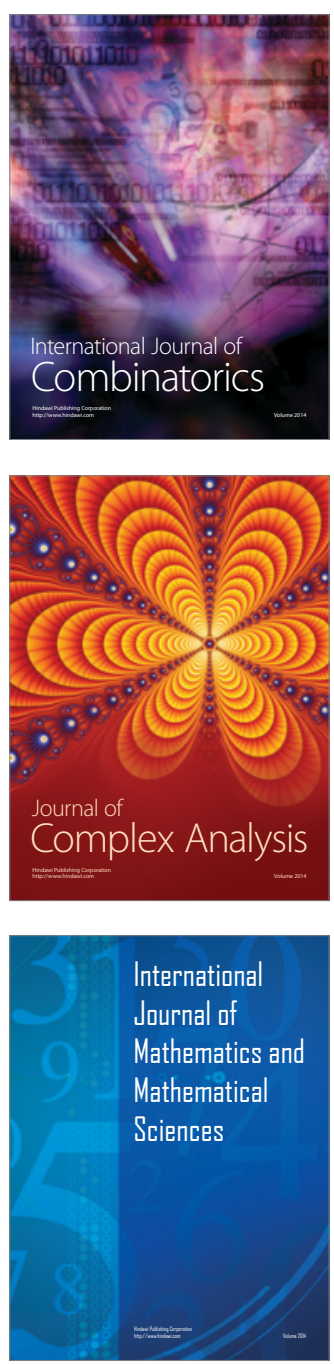
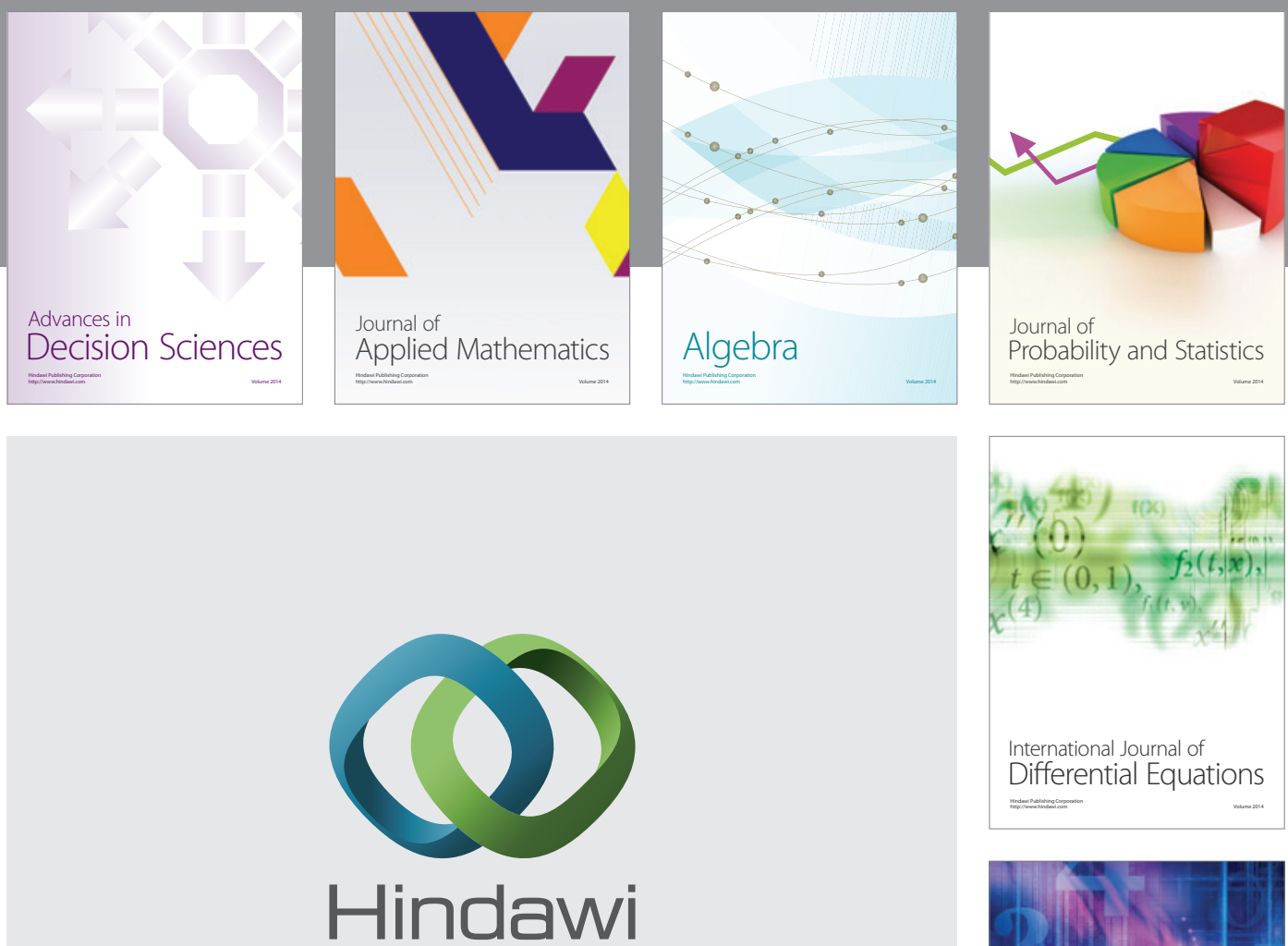

Submit your manuscripts at http://www.hindawi.com
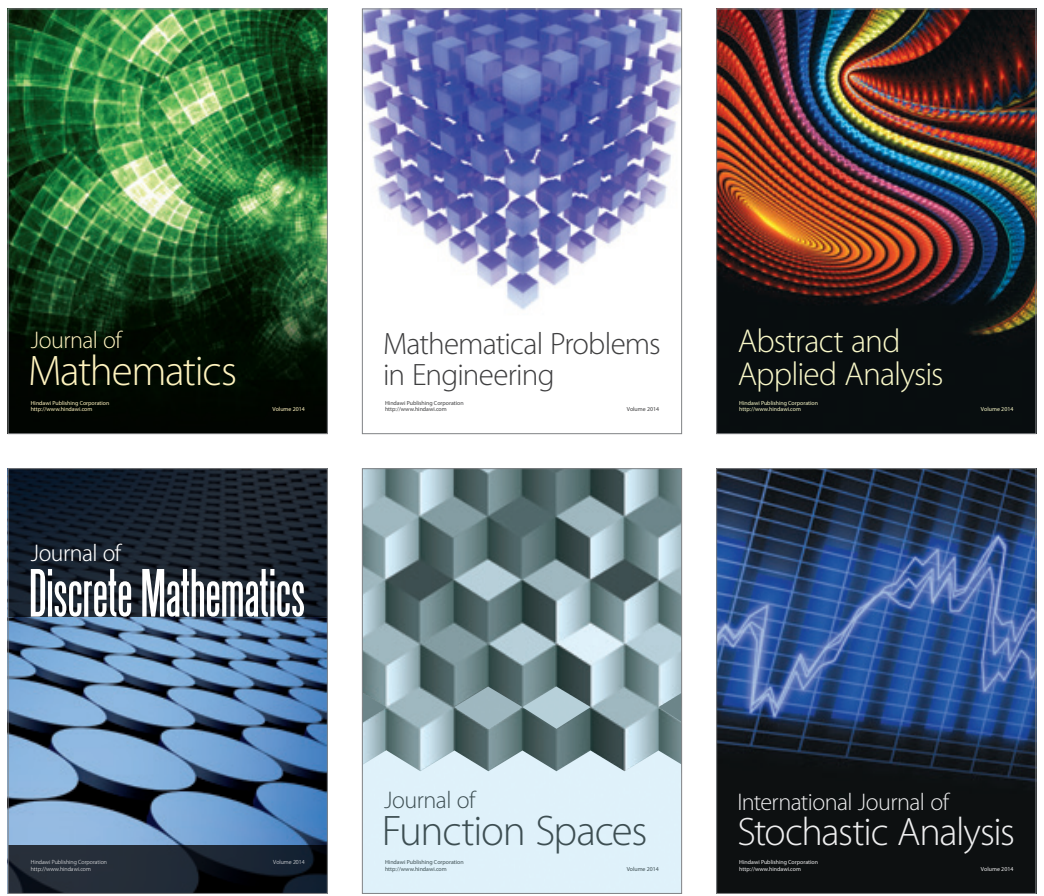

Journal of

Function Spaces

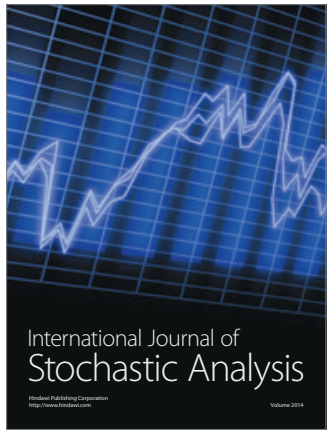

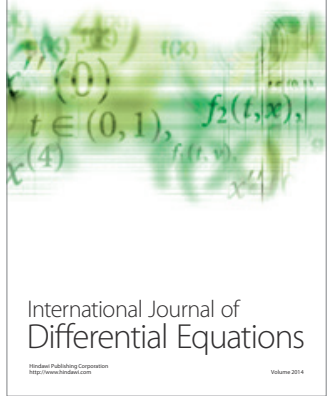
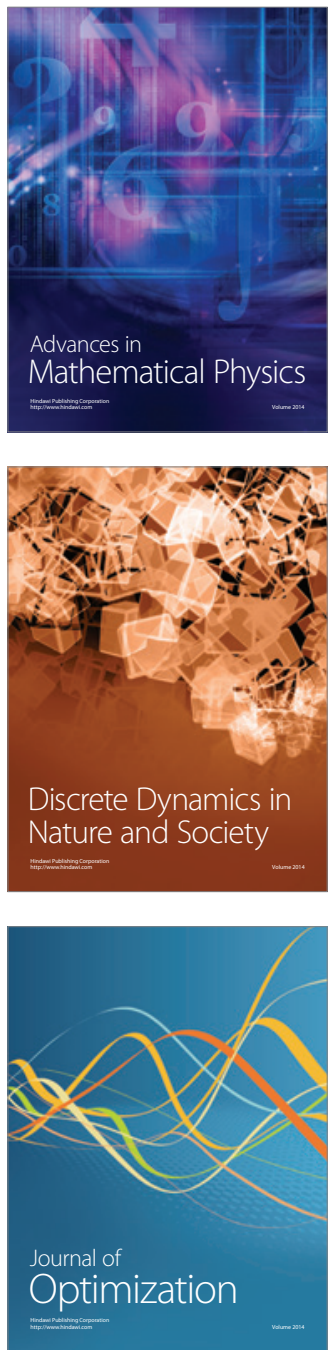